\title{
圆 \\ Occurrence, clinical manifestation and the epidemiological implications of naturally occurring canine trypanosomosis in western Kenya
}

\section{G.O. MATETE*}

Kenya Trypanosomiasis Research Institute P.O. Box 362, Kikuyu, Kenya

\begin{abstract}
MATETE, G.O. 2003. Occurrence, clinical manifestation and the epidemiological implications of naturally occurring canine trypanosomosis in western Kenya. Onderstepoort Journal of Veterinary Research, 70:317-323

Domestic dogs were screened for Trypanosoma brucei infection using the haematocrit centrifugation technique as part of routine active surveillance exercises in the Busia and Teso districts of Kenya. The purpose was to assess the role of dogs as sentinels for the occurrence of human sleeping sickness. Out of 200 dogs screened, five were found to be infected at the various test sites. These five succumbed to the disease within four weeks, and exhibited a distinct and pronounced corneal opacity before death. Blood from two naturally infected dogs were tested for the presence of the serum resistance associated (SRA) gene and one tested positive, confirming it as human infective ( $T$. brucei rhodesiense) prevalence $(0.5 \%)$. It is considered that the occurrence of this clinical sign could be used as an early warning prediction of future outbreaks. This type of prediction could form an integral part of an indigenous technical knowledge set in areas lying at the edges of the tsetse (Glossina) belts where $T$. brucei is the main trypanosome species that affects dogs. The occurrence of corneal opacity in dogs could indicate a rise in the levels of $T$. brucei a proportion of which could be human infective $T$. b. rhodesiense circulating in the population early enough before disease outbreak occurs. It is thought that during sleeping sickness epidemics the domestic dog will be the first casualty rapidly succumbing to disease long before it is noticed in man. Prompt prediction of disease outbreaks would thus enable early interventions that would reduce the morbidity, mortality and the general economic losses associated with sleeping sickness to be instituted.
\end{abstract}

Keywords: Canine, epidemiology, sleeping sickness, trypanosomosis

\section{INTRODUCTION}

Human trypanosomosis occurs in 36 African counties that lie between latitudes $14^{\circ}$ North and $29^{\circ}$ South. Western Kenya is an area endemic for human trypanosomosis (sleeping sickness), which is a cause for public health concern wherever it occurs. Current estimates of the parasitological prevalence of Trypanosoma brucei in animals in west-

\footnotetext{
* E-mail: georgematete2002@yahoo.com

Accepted for publication 24 June 2003-Editor
}

ern Kenya stand between $2 \%$ and $5 \%$ but may rise beyond $10 \%$ during outbreaks with three to five cases of sleeping sickness in humans (Matete, unpublished data 2002). The disease prevalence, however, increases beyond $70 \%$ during epidemics involving over 10 sleeping sickness cases (Welburn, Picozzi, Fevre, Coleman, Oditt, Carrington \& Maudlin 2001).

Trypanosomosis of dogs was first described in 1908 (Bevan 1913). It has been established that dogs are susceptible to various trypanosomes including T. brucei brucei, T. brucei rhodesiense, $T$. brucei gambiense, Trypanosoma congolense, Tryp- 
anosoma evansi and Trypanosoma cruzi (Morrison, Murray, Sayer \& Preston 1983). The clinical signs of disease have been described by Morrison et al. (1983) and Kaggwa, Munyua \& Mugera (1983; 1984), and the pathology by Kaggwa, Munyua \& Mugera (1988). Gibson \& Gashumba (1983) described dogs as carriers of $T$. b. rhodesiense in Uganda. In Nigeria, trypanosomosis in dogs is a common occurrence in the T. b. gambiense belt, where Omamegbe, Orajaka \& Omehelu (1984) reported the ocular, lymphoadenopathic and meningial forms as the three distinct forms of disease. Dogs are an integral part of the lives of livestock farmers in western Kenya, an endemic area for $T$. brucei rhodesiense. Blind dogs that eventually die within a few weeks of the start of clinical signs have been associated with potential outbreaks of sleeping sickness in humans by the local communities living within the tsetse belt of Kenya and Uganda. They therefore bring their dogs for screening during active surveillance programmes (Oditt, personal communication 2000). Transmission mainly occurs around the watering sites and is sustained in animal-tsetse-animal cycle by Glossina pallidipes. The infection is then spread to humans by Glossina fuscipes fuscipes.

Efforts to utilize current markers, like minisatellites, have been able to distinguish between Ugandan $T$. b. rhodesiense and T. b. brucei (Macleod, Twedie, Welburn, Maudlin, Turner \& Tait 2000). The ectopic expression of human serum resistance (SRA), a 743 bp-molecular mass gene product allows $T$. $b$. rhodesiense to survive exposure to normal human serum. The presence of the gene product correlates to human serum resistance and offers the prospect of a simple one-step diagnostic kit for $T$. $b$. rhodesiense (Xong, Vanhamme, Chamekh, Chifwembe, Van den Abbeele, Pays, Van Meirvene, Hamers, De Baetselier \& Pays 1998; Welburn et al. 2001). The screening of the SRA gene has been shown to distinguish between $T$. b. rhodesiense and T. b. brucei in non-human hosts (Welburn et al. 2001).

Treatment of dogs is not always successful, and break-through infection after isometamidium prohylaxis occurs in some (Lopes-Pereira, Lopes-Pereira, Escrivao, Schwalbach \& Pinto 1997). Melarsoprol $\AA^{\circledR}$ (Aventis Pharma) is the drug of choice in the chemotherapeutic management of the late stage- $T$. $b$. rhodesiense sleeping sickness in humans. A 10day treatment protocol has been investigated for $T$. b. gambiense in Angola, and it is hoped that this will reduce the duration of confinement from 42 days to just below 14 days (Blum \& Burri 2002). However, there are no reports on the use of Melarsoprol ${ }^{\circledR}$ in dogs. In the current study a short-course treatment with Melarsoprol ${ }^{\circledR}$ was attempted on one naturally infected dog. The occurrence, clinical manifestations and epidemiological implications of canine trypanosomosis in $T$. b. rhodesiense sleeping sickness endemic areas are discussed in detail. An attempt was also made to validate dogs as carriers of $T$. b. rhodesiense by testing their infective trypanosomes for the presence of the SRA gene.

\section{MATERIAL AND METHODS}

The study was undertaken in Teso and Busia districts that fall within the Lake Victoria basin of western Kenya. These districts cover an area of 1819 $\mathrm{km}^{2}$ and their altitude ranges from $1128 \mathrm{~m}$ in the extreme south to $1463 \mathrm{~m}$ above sea level in central and north Teso. The districts have a mean annual rainfall of approximately $1270 \mathrm{~mm}$, which is bimodal in distribution. Maximum temperatures range between $26{ }^{\circ} \mathrm{C}$ and $30^{\circ} \mathrm{C}$ while the mean minimum temperature range between $14{ }^{\circ} \mathrm{C}$ and $22^{\circ} \mathrm{C}$. The tsetse fly species present in the area are G. f. fuscipes and G. pallidipes in riverine and savannah habitats, respectively. Most of the uncultivated land is presently encroached by Lantana camara and Digithonia spp. thickets that form good breeding grounds for tsetse flies.

The investigation was carried out over a 1-year period, between April 2001 and March 2002, as part of a regular active surveillance programme. The administrative locational/sub-locational maps were used to select locations for purposive samplings based on the previous occurrence of sleeping sickness and reports of deaths in cattle. After site selection, a double randomisation was done from a complete list of villages within the locations to ensure an even distribution of sampling sites. A geographical positioning system (GPS) was used to mark the exact locations of blind dogs. These sites were Obochun, Koruruma, Busia township, Budubusi and Rwambwa. A questionnaire was used to determine the historical features of the disease. At the same time humans and cattle were also screened, and for comparative purposes, the apparent density of tsetse flies was determined.

Five methods were used to detect trypanosomes in the blood of dogs, which was collected from the jugular vein. These were the microscopic examination of wet smears and Giemsa-stained thin blood smears, the haematocrit centrifugation technique (Woo 1969), buffy coat examination (Murray, Mur- 
ray \& Mclntyre 1977) and a spinal tap for cerebrospinal fluid (CSF). Haematological analysis for packed cell volume and haemoglobin concentration was done from blood drawn into capillary tubes. Examination of the morphological features of trypanosomes was made using the Giemsa-stained thin smears, and their motility in wet preparations. Parasites were then subjected to blood incubation and an infectivity test to determine their infectivity for humans (Rickman \& Robson 1970; Brun \& Jenni 1987). One dog, whose owner consented to treatment with a 10-day course of Melarsoprol ${ }^{\circledR}$ at 2.2 $\mathrm{mg} / \mathrm{kg}$ body mass, was treated intravenously at 9:00 daily though either the radial or femoral veins. The dog was subsequently euthanased 1 week after the termination of treatment on the instructions of the owner and an extensive postmortem examination was carried out. Samples from the eyes and brain were taken and preserved in $10 \%$ formalin, and tissue sections cut, stained with haematoxylin and eosin according to routine techniques. During the necropsy, impression smears were made from the cerebellum and stained with Giemsa.

The polymerase chain reaction $(\mathrm{PCR})$ and SRA gene probes were used to test for presence of $T$. $b$. rhodesiense in two dogs from the two sleeping sickness focus of Kenya. The two isolates compared were KETRI 3119 isolated from South Nyanza and KETRI 3739 isolated at Obochun in Teso district in 2001. Samples were prepared for standard PCR cycling and screening for presence of $T$. brucei sl., was done as described by De Greef, Inberrechts, Matthyssens, Van Meirvenne \& Hamers (1989) and Artama, Agey, \& Donelson (1992), and SRA gene detection was done according to Gibson, Backhouse \& Griffiths (2002).

\section{RESULTS}

A total of 200 dogs of indeterminate breed were sampled during the study of which five were positive for $T$. brucei, giving a total prevalence of $2.5 \%$. Four dogs $(2.0 \%)$ had T. b. brucei, while T. b. rhodesiense was present in one (0.5\%). The comparative infection rate in man was estimated at $0.5 \%$, in cattle $10.2 \%$ and $0.2 \%$ in tsetse flies. Most respondents to the questionnaire indicated that dogs had died after becoming blind, with conservative mortality estimated at $34 \%$ within the previous three years. A variety of non-specific clinical signs were noted in the five infected dogs. These included depression, sleepiness, pale mucous membranes, lethargy, anorexia, mass loss and a rough hair coat. Other signs were recumbence, enlarged superficial lymph nodes, dyspnoea and fever with a mean of $39.8^{\circ} \mathrm{C}$. Neurological observations included dullness and lack of coordination manifesting as paraplegia, bilateral hyperflexic patellar reflexes, lack of conscious proprioception in the pelvic limbs and marginally depressed hopping reflexes of the forelimb. Corneal opacity, conjunctivitis with a mucopurrulent discharge, uveitis, haemorrhage and turbidity in the anterior chamber, and loss of vision were also noted. However, the predominant sign was varying degrees of bilateral cloudiness of the cornea due to the turbid aqueous humour (Fig. 1).

Oedema of the front limbs, head and neck was also observed. Progressive mental deterioration similar to the aggressive behaviour associated with rabies, together with lethargy and mania, were the historical observations obtained from the questionnaire. Wet blood films in the five infected dogs were teeming with trypanosomes and haematology indicated a leukocytosis as a result of neutrophilia and lymphocytosis. Moderate normocytic normochromic anaemia with reduced packed cell volume, haemoglobin values and red blood cells (RBC) counts were also observed. The cerebrospinal fluid of the one dog was still positive for trypanosomes, as were the brain impression smears. The numbers of lymphocytes in the CSF were elevated. Following treatment of one dog with Melarsoprol ${ }^{\circledR}$, trypanosomes disappeared in blood within $24 \mathrm{~h}$. In 5 days a gradual but progressive clearing of the uvea was observed, which was followed by a complete recovery of the eyesight by 10 days after the first treatment (Fig. 2).

The dog gained in strength, began to feed and became more active. Although it responded clinically and trypanosomes were absent in the CSF, they were later found in the brain impression smears. The dog was then destroyed at day 17. At necropsy the body was slightly emaciated due to anorexia, evidenced by prominence of dorsal vertebral and scapular spines. In addition the dog exhibited moderate cerebral meningitis and oedema, and scattered haemorrhages were present in the meninges. Cellular infiltration of the leptomeninges with macrophages, neutrophils, plasma cells and lymphocytes was observed. Moderate encephalitis manifested by the presence of perivacular cuffing, which was particularly prominent in the brain stem and cerebellum, was evident. Astrocyte and meningial hyperplasia was also present. The front limbs were oedematous with a muco-purulent discharge at the radio-ulnar junction on the left. These were presumed to be septic decubitus wounds due 


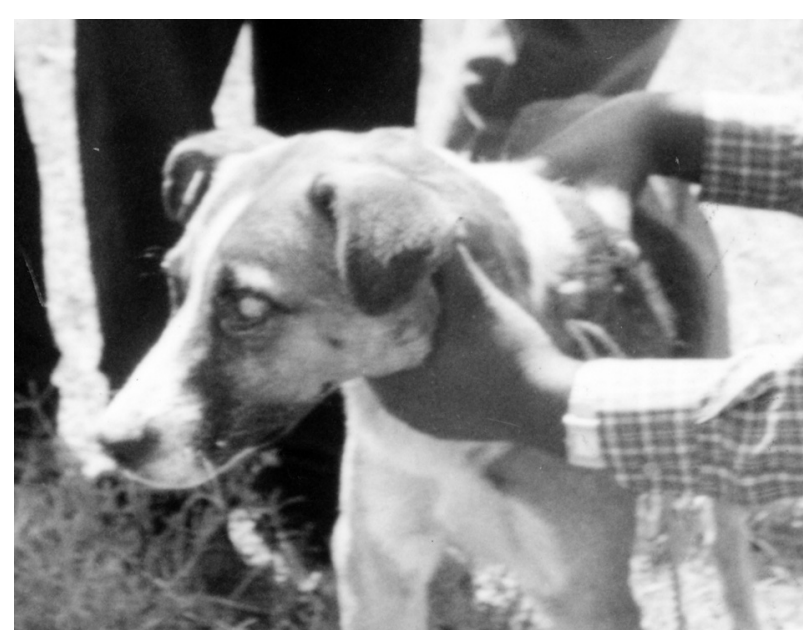

FIG. 1 Naturally infected dog showing corneal opacity

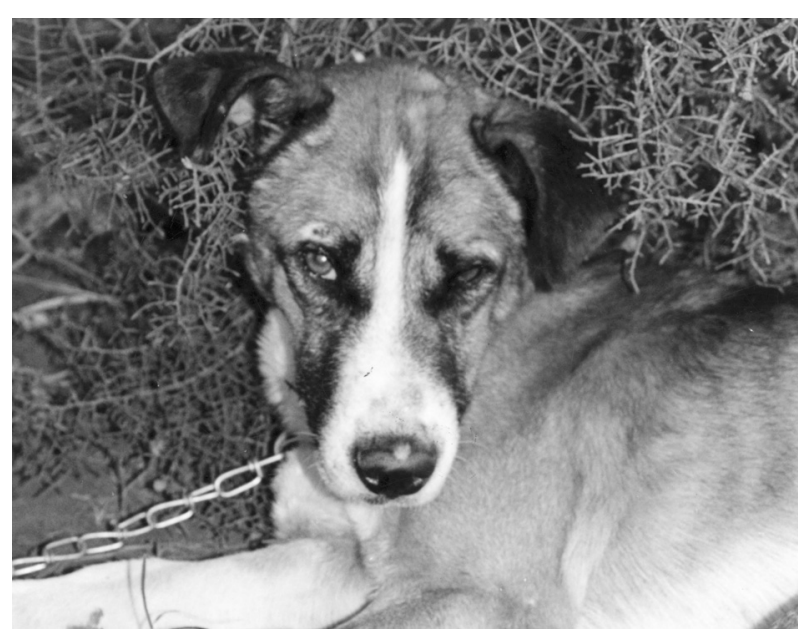

FIG. 2 The same dog as in Fig. 1 after complete recovery of the eyesight

\begin{tabular}{|c|c|c|}
\hline T. b. brucei & $\mathrm{R}_{0}$ & T. b. rhodesiense \\
\hline 1 & & \\
\hline $100 \%$ & $50 \%$ & $100 \%$ \\
\hline Endemic (2-5 \%) & $0 \%$ & Epidemic (> $70 \%$ ) \\
\hline
\end{tabular}

$\mathrm{R}_{0}$ Risk of transmission for sleeping sickness

FIG. 3 Model of the prevailing situation of trypanosomiasis in western Kenya. When the prevailing levels of $T$. b. rhodesiense exceed $10 \%$ they undergo a clonal expansion to induce an outbreak or even epidemic. The same situation can arise when T. brucei exceed $50 \%$ in a population, then a switch to $T$. b. rhodesiense occurs and these then undergo a clonal expansion

TABLE 1 Response of dog stabilates to BIIT and SRA gene probe

\begin{tabular}{|l|l|l|l|l|}
\hline Number & Date isolated & BIIT & PCR (T. brucei sl.) & SRA gene \\
\hline KETRI 3738 & $5 / 11 / 01$ & + & + & $+^{*}$ \\
KETRI 37391 & $31 / 5 / 01$ & + & + & $+^{*}$ \\
KETRI 31192 & South Nyanza & - & + & $-^{*}$ \\
\hline
\end{tabular}

Key

1 Isolate 3739 was a sub culture of 3738 passages in mice; SRA gene were shown to be conserved

2 Isolate was from Lambwe valley a different focus of sleeping sickness

* Isolates tested by PCR and by SRA gene probes few due to cost

All five dogs were BIIT positive 
to long-standing sleepiness. About $50 \mathrm{~m} \ell$ of strawcoloured fluid occurred in the abdomen and hydrothorax was also observed.

Polymerase chain reaction was able to identify the two isolates as being T. brucei sl. The SRA gene probe differentiated KETRI 3119 as T. b. brucei and KETRI 3739 as $T$. b. rhodesiense. The isolate KETRI 3739 was also resistant to human serum and multiplied when inoculated in mice after incubation in normal human serum (Table 1.).

\section{DISCUSSION}

Although it has been established that dogs are carriers of $T$. b. rhodesiense data remains inadequate due to the use of techniques of low sensitivity. The infection rate estimated at $0.5 \%$ in man, $10.2 \%$ in cattle and $0.2 \%$ in tsetse in the current study compared well with the finding in the Busoga focus of $0.6 \%$ in man, $10.5 \%$ in cattle and $0.2 \%$ in tsetse flies reported by Maudlin, Welburn, Gashumba, Okuna \& Kalunda (1990). In an endemic situation as evidenced in western Kenya human infective trypanosomes ( $T$. b. rhodesiense) are difficult to detect due to their low prevalence. Macleod, Tait \& Turner (2001) have already shown that a clonal expansion of $T$. $b$ rhodesiense occurs in the field. This could be an important determinant of trypanosome diversity except where clones have acquired the ability to infect humans. They report that when $T$. b. brucei acquires ability to infect humans it then spreads rapidly through the human population from the single source event. It is now postulated that tsetse flies feed on dogs at levels approximate to the $9 \%$ observed in cattle and that human infective trypanosomes circulate in tsetse at sub infective doses within the endemic areas. This is in agreement with Anon (1991), that animal sentinel systems, particularly dogs, have been used as surrogates to human exposure to environmental risks or to monitor complex environments. An outbreak of sleeping sickness would thus occur when either the prevalence of $T$. b. rhodesiense is at least $10 \%$, or rapidly transforms itself into an epidemic when the prevalence exceeds $50 \%$. The same situation could arise when proportion of $T$. b. brucei circulating in the population is $50 \%$. This is sufficient to enable a switch between human serum sensitive and human serum resistant forms in adequate numbers to cause an outbreak. The findings of Rickman \& Kolala (1980), De Greef et al. (1989) and Macleod et al. (2001) are consistent with the postulates of this model. An extensive study using molecular techniques may provide confirmation to the scenario suggested (Fig. 3).
It was clear from this model that time and space were important determinants of both the genetic diversity of trypanosomes circulating in the area, the course of the disease and its final outcome. The clinical signs and pathology observed in the current study were similar to those described by Kaggwa et al. (1983; 1984) and Morrison et al. (1983) for both experimental and natural infections. The predominant clinical sign in all the cases was the blue/white cloudiness of the cornea in all the dogs. This prevalence of corneal opacity of $2.5 \%$ in $T$. b. rhodesiense sleeping sickness areas differs from the $52.8 \%$ obtained by Omamegbe et al. (1984) in T. b. gambiense areas of Nigeria. The description is similar to that described by Sayer, Morrison, Preston, Hird, Price \& Murray (1979). The opacity was bilateral in all the dogs studied. Corneal opacity is, however, rarely noted in humans (Apted 1970), and is attributed to the descent of parasites along the optic artery from the brain with the subsequent induction of immune complexes into the aqueous humour. These complexes form granular deposits within the aqueous, which progressively cloud the cornea by adhering to its inner surface and thus hinder vision. This is in agreement with one of the hypotheses of the immune reaction described by Lambert, Berney \& Kazyumba (1981). Other presenting signs, such as oedema of the limbs, emaciation, loss of muscle mass, hydrothorax and ascites, were similar to those described by Morrison et al. (1983).

The nervous signs exhibited by the dogs were similar to those noted by Berger, Palmer, Hodges \& Hall (1991) in infections of $T$. cruzi. These changes in behaviour could be attributed to meningial invasion with subsequent inflammation and oedema. The invasion is induced by proteases such as trypanopains, oligopeptidases and cell surface associated acid phosphates produced by the parasites (Lonsdale-Eccles \& Grab 2002). These result in a host immune response with a large numbers of lymphocytes and plasma cells forming immune complexes of IgM, morula cells and T-helper cells within the brain (Zola, Wassuoumbou-Loubienga, Goma \& Mouanga-Yidika 1994). Brain impression smears in this study indicated localization of trypanosomes in the brain, which was similar to findings of Ikede \& Losos (1972), and is suggested as the cause of most treatment failures. The changes noted in the cerebrospinal fluid were an indication of a non-suppurative inflammation similar to that observed in T. cruzi by Berger et al. (1991).

The pronounced corneal opacity in the dog could be used as a preliminary sentinel sign for the transmission of future sleeping sickness outbreaks as 
part of the indigenous technical knowledge. In areas where the occurrence of $T$. vivax predominates over that of $T$. congolense, $T$. brucei becomes the only causative agent for trypanosomosis in dogs. The disease in dogs within such areas may therefore be attributed to a rise in the circulating levels of $T$. bru$c e i$ in the animal population. There is circumstantial evidence that $T$. brucei in dogs could therefore be useful in predicting the occurrence of sleeping sickness outbreaks in western Kenya. The sporadic and very low disease prevalence in dogs within the area closely reflects the situation occurring in humans. Dogs have similarly been used as sentinel animals to monitor the transmission of $T$. cruzi in rural areas in Argentina (Castanera, Lauricella, Chuit \& Gurtler 1998). When dogs are used as sentinel animals, the occurrence of corneal opacity would indicate a rise in levels of $T$. brucei circulating in the population. If molecular tools, e.g. SRA gene probes, are then used to confirm the parasites in dogs as $T$. $b$. rhodesiense, sleeping sickness will be detected before outbreaks occur in humans. The dog may in itself have little relevance as a reservoir for the human infective forms of trypanosomes considering the relatively acute phase of the disease with death occurring between 2 weeks and 1 month after the onset of clinical signs. The clinical signs manifest more rapidly that it does in man where it is reported to take between 3 weeks and 6 months (Odiit, Kansiime \& Enyaru 1997). The finding of the SRA gene in trypanosomes infecting a dog in this study confirms the ability of dogs to carry the human infective trypanosomes. It is noteworthy that the isolate from Kenya had been obtained from Obochun, an area that neighbours the most recent site of an outbreak of sleeping sickness in Uganda (Unpublished LIRI reports 2001). Since T. b. brucei is able to switch from human serum sensitive to human serum resistant forms, the course of the disease in the dog should therefore be studied from the beginning of outbreaks to the epidemic phase to see if this hypothesis holds. Prompt prediction of disease outbreaks would thus enable early interventions to be instituted that would reduce the morbidity and mortality of sleeping sickness as well as the general economic losses associated with it.

Complete recovery of sight was obtained in ten days compared to reports of 45 days in $T$. evansi infections as described by Vershney, Varsheny \& Dwivedi (1998). It is possible that with the cure of trypanosomes, the opacity could then resolve spontaneously. Further tests are required to validate the use of Melarsoprol ${ }^{\circledR}$ in the treatment of valuable dogs, e.g. bomb or drug sniffers and guard dogs with trypanosomosis.

\section{ACKNOWLEDGEMENTS}

I am grateful for the excellent technical assistance by staff of KETRI Alupe centre. I also wish to thank my colleagues, Dr Nyamwaro and Dr Makumi, who read the manuscript. This investigation received support from the European Union through the FITCA Regional fund. This work is published with permission of the Director of KETRI.

\section{REFERENCES}

ANON. 1991. Animals as sentinels of environmental health hazards. Washington, DC: National Academy Press.

ARTAMA, W.T., AGEY, M.W. \& DONELSON, J.E. 1992. DNA comparison of Trypanosoma evansi (Indonesia) and Trypanosoma brucei spp. Parasitology, 104:67-74.

APTED, F.I.C. 1970. Clinical manifestation and diagnosis of sleeping sickness, in The African Trypanosomiasis, edited by H.W. Mulligan. London: George Allen and Unwin.

BERGER, S.L., PALMER, R.H., HODGES, C.C., \& HALL, D.G. 1991. Neurologic manifestations of trypanosomiasis in a dog. Journal of the American Veterinary Medical Association, 198:132-134.

BEVAN, E.W. 1913. Preliminary notes on a trypanosome causing disease in man and animal in the Sebungwe district of southern Rhodesia. Journal of Tropical Medicine and Hygiene, 16:113-117.

BLUM, J. \& BURRI, C. 2002. Treatment of late stage sleeping sickness caused by T. b. gambiense: a new approach to the use of an old drug. Swiss Medical Weekly, 132:51-56.

BRUN, R. \& JENNI, L. 1987. Human serum resistance of metacyclic forms of Trypanosoma brucei brucei, T. brucei rhodesiense and T. gambiense. Parasitology Research, 73:218223.

CASTANERA, M.B., LAURICELLA, M.A., CHUIT, R. \& GURTLER, R.E. 1998. Evaluation of dogs as sentinels of the transmission of Trypanosoma cruzi in a rural area of northwestern Argentina. Annals of Tropical Medicine and Parasitology, 92:671-683.

DE GREEF, C., IMBERECHTS, H., MATTYSSENS, G., VAN MEIRVENNE, N. \& HAMERS, R. 1989. A gene expressed only in serum resistant variants of Trypanosoma brucei rhodesiense. Molecular and Biochemical Parasitology, 36:69176.

GIBSON, W., BACKHOUSE, T. \& GRIFFITHS, A. 2002. The human serum resistance associated gene is ubiquitous and conserved in Trypanosoma brucei rhodesiense throughout East Africa. Infection, Genetics and Evolution, 25:1-8

GIBSON, W. \& GASHUMBA, J.K. 1983. Isoenzyeme characterization of some trypanosome stocks from a recent trypanosomiasis epidemic in Uganda. Transactions of the Royal Society of Tropical Medicine and Hygiene, 77:114118.

IKEDE, B.O. \& LOSOS, G.J. 1972. Spontaneous canine trypanosomiasis caused by $T$. brucei: meningo-encephalomyelitis with extravascular localization of trypanosomes in the brain. Bulletin of Epizootic Diseases in Africa, 120:221-8.

KAGGWA, E., MUNYUA, W.K. \& MUGERA, G.M. 1983. Pathogenicity of Trypanosoma brucei brucei in dogs. Bulletin of Animal Health and Production in Africa, 32:360-368.

KAGGWA, E., MUNYUA, W.K. \& MUGERA, G.M. 1984. The pathology of Trypanosoma brucei infection in the dog. Bul- 
letin of Animal Health and Production in Africa, 33:69-75.

KAGGWA, E., MUNYUA, W.K. \& MUGERA, G.M. 1988. Relapses in dogs experimentally infected with Trypanosoma brucei and treated with diminazene aceturate and isometamidium chloride. Veterinary Parasitology, 27:199-208.

LAMBERT, P.H., BERNEY, M. \& KAZYUMBA, G. 1981. Immune complexes in serum and cerebrospinal fluid in African trypanosomiasis. Correlation with polyclonal B cell activation and with intracerebral immunoglobulin synthesis. Journal of Clinical Investigation, 67:77-85.

LONSDALE-ECCLES, J.D. \& GRAB, D.J. 2002. Trypanosome hydrolases and the blood-brain barrier. Trends in Parasitology, 18:17-19.

LOPES-PEREIRA, C.M., LOPES-PEREIRA, D., ESCRIVAO, R.A., SCHWALBACH, L.J. \& PINTO, F.G. 1997. Chemoprophylaxis of trypanosomosis in dogs. Proceeding of the International Scientific Council of Trypanosomiasis Research and Control, 24 $4^{\text {th }}$ meeting, Maputo, Mozambique.

MACLEOD, A., TAIT, A. \& TURNER, C.M.C. 2001. The population genetics of Trypanosoma brucei and the origin of human infectivity. Philosophical Transactions of the Royal Society of London, Biological Sciences, 356:1035-1044.

MACLEOD, A., TWEDIE, A., WELBURN, S.C., MAUDLIN, I., TURNER, C.M.C., \& TAIT, A. 2000. Minisatellite marker analysis of Trypanosoma brucei: Reconciliation of clonal, panmitic and endemic population genetic structures. Proceedings of the National Academy of Sciences of the United States of America, 96:13442-13447.

MAUDLIN, I., WELBURN, S.C., GASHUMBA, J.K., OKUNA, N.S. \& KALUNDA, M. 1990. The role of cattle in the epidemiology of sleeping sickness in Uganda. Bulletin for the Society of French Parasitologists, 8:788.

MORRISON, W.I., MURRAY, M., SAWYER, P.D. \& PRESTON, J.M. 1983. The pathogenesis of experimentally induced Trypanosoma brucei infection in the dog. 1. Tissue and Organ damage. American Journal of Pathology, 102:168181.

MURRAY, M., MURRAY, P.I.C. \& MCINTYRE, W.I.M. 1977. Improved parasitological technique for diagnosis of African trypanosomiasis. Transactions of the Royal Society of Tropical Medicine and Hygiene, 71: 325-326.

ODIIT, M., KANSIIME F., ENYARU J.C.K. 1997. Duration of symptoms and case fatality of sleeping sickness caused by
Trypanosoma brucei rhodesiense in Tororo, Uganda. East African Medical Journal, 74: 792-795.

OMAMEGBE, J.O., ORAJAKA, L.J.E. \& OMEHELU, C.O. 1984. The incidence and clinical forms of naturally occurring canine trypanosomiasis in two veterinary clinics in an Anambra State of Nigeria. Bulletin of Animal Health and Production in Africa, 32:23-29.

RICKMAN, L.R., \& KOLALA F. 1980. Relationship between Trypanosoma brucei and T. rhodesiense. Transactions of the Royal Society of Tropical Medicine and Hygiene, 74: 817-819.

RICKMAN, L.R., \& ROBSON, J. 1970. The blood incubation infectivity test: a simple test, which may serve to distinguish Trypanosoma brucei from $T$. rhodesiense. Bulletin of the World Health Organisation, 42:650-651.

SAYER, P.D., MORRISON, W.I., PRESTON, J.M., HIRD, S.F., PRICE, J.E. \& MURRAY, M. 1979. African trypanosomiasis in the dog. Proceedings of the International Scientific Council of Trypanosomiasis Research and Control, $15^{\text {th }}$ meeting, Gambia, 489-496.

VERSHNEY, J.P., VARSHNEY V.P. \& DWIVEDI, S.K. 1998. Clinico-endocrinological findings in clinical trypanosomosis in dog. Journal of Veterinary Parasitology, 12:143-144. (Abstract in PUBMED).

WELBURN, S.C., PICOZZI, K., FEVRE, E.M., COLEMAN, P.G., ODITT, M., CARRINGTON, M. \& MAUDLIN, I. 2001. Identification of human-infective trypanosomes in animal reservoir of sleeping sickness in Uganda by means of serum resistance associated (SRA) gene. Lancet, 358:2017-19.

WOO, P.K.T., 1969. The haematocrit centrifugation for the detection of trypanosomes in blood. Canadian Journal of Zoology, 47:921-923.

XONG, H., VANHAMME, L., CHAMEKH, M., CHIFWEMBE, C.E., VAN DEN ABBEELE, J., PAYS, A., VAN MEIRVENE, N., HAMERS, R., DE BAETSELIER, P. \& PAYS, E. 1998. A VSG expression site-associated gene confers resistance to human serum in Trypanosoma rhodesiense. Cell, 95:839846.

ZOLA, J.M., WASSUOUMBOU-LOUBIENGA, S., GOMA, G.C. \& MOUANGA-YIDIKA, G. 1994. Meningio-encephalitite aigue a Trypanosoma brucei gambiense revelee par un Oedeme papillaire. Bulletin of Society of Pathology and Exobiology, 87:312-314. 